\title{
The performance evaluation of IEEE 802.11 against IEEE 802.15.4 with low transmission power
}

\begin{abstract}
Theoretically, IEEE 802.15.4 is more suitable for resource-constraint ad-hoc network because of its low power consumption characteristic. However, performance of IEEE 802.11 is unknown if its transmission power and receiver sensitivity are limited to match those levels of IEEE 802.15.4. In this paper, the performance evaluation of IEEE 802.11 against IEEE 802.15.4 with those power levels is presented. The transmission power and receiver sensitivity of IEEE 802.11 are derived from IEEE 802.15.4 in order to compare their performance and energy consumption. The Ad-hoc On-Demand Distance Vector (AODV) and Dynamic Source Routing (DSR) routing protocols are exploited for the evaluation of varying network size and node placement models, namely Grid, Random and Uniform. Simulation results show that IEEE 802.11 still outperforms IEEE 802.15.4 in terms of packet delivery ratio, throughput, average end-to-end delay (latency) and average jitter. Besides, the average energy consumed by each node in IEEE 802.11 is even lower than that in IEEE 802.15.4. Nevertheless, under the limited energy condition, the transmission range of IEEE 802.11 is far lower than that supported by IEEE 802.15.4. This is a trade-off of IEEE 802.11 compared to IEEE 802.15.14.
\end{abstract}

Keyword: IEEE 802.11; IEEE 802.15.4; AODV; DSR; Low power; Ad-hoc network 